\title{
Sugars in carbonaceous chondrites
}

\author{
YOSHIHIRO FURUKAWA ${ }^{1^{*}}$, YOSHITO \\ CHIKARAISHI $^{2}$, NAOHIKO OHKOUCHI ${ }^{3}$, NANAKO O. \\ OGAWA $^{3}$, DANIEL P. GLAVIN ${ }^{4}$, JASON P. \\ DWORKIN $^{4}$, CHIAKI ABE ${ }^{1}$, AND TOMOKI NAKAMURA ${ }^{1}$
}

${ }^{1}$ Department of Earth Science, Tohoku Univeristy, Sendai, Japan (*Correspondance: furukawa@tohoku.ac.jp)

${ }^{2}$ Institute for Low Temperature Sciecne, Hokkaido

Univeristy, Sapporo, Japan

${ }_{3}^{3}$ Japan Agency for Marine-Earth Science and Technology, Yokosuka, Japan

${ }^{4}$ NASA Goddard Space Flight Center, MD, USA

Sugar is a major building block of life working in many steps in biological reactions. Ribose is an especially important sugar as a component of RNA which works in essential processes of all living biota. Amino acids and nucleobases, other major building blocks of life, have been found in carbonaceous chondrites [e.g., 1,2]. Extraterrestrial sugars have also been investigated in meteorites for long time and a small sugar molecule was found [3]. However, the presence of extraterrestrial bio-essential sugar remained unclear due to its difficulty in analysis.

We investigated sugars in extracts of several meteorites using gas chromatography mass spectrometer after a derivatization and found several sugars including ribose, arabinose, xylose, and lyxose from Murchison and NWA 801 meteorites [4]. Some of these sugar molecules have distinct stable carbon isotope ratio from terrestrial biological sugars, indicating those extraterrestrial origin. Murchison and NWA 7020 meteorite have experienced significant aqueous processes in those parent bodies, whereas the aqueous processes were significantly limited for NWA 801. The abundance of these sugars is consistent with the abundance of the sugars in the product of formose reaction in which formaldehyde and glycolaldehyde react each other to form sugars in alkaline solution, suggesting that the detected sugars in meteorites were formed from formose-like presses before and/or after the aqueous precedes of the meteorite parent bodies.

These results suggest that meteorites have introduced extraterrestrial bio-essential sugars from asteroids to inner planets including prebiotic Earth.

References: [1] Glavin et al., Meteorit. Planet. Sci. 45, 1948-1972 (2011). [2] Callahan et al., PNAS 108, 1399513998 (2011). [3] Cooper et al., Nature 414, 879-883 (2001). [4] Furukawa et al., PNAS 116, 24440-24445 (2019). 